Mappemonde

Revue trimestrielle sur l'image géographique et les formes du territoire

$120 \mid 2017$

Varia

\title{
Le palmarès 2016 du concours Géovisualisation et cartographie dynamique
}

Anne-Christine Bronner

(2) OpenEdition

Journals

Édition électronique

URL : http://journals.openedition.org/mappemonde/3166

DOI : 10.4000/mappemonde.3166

ISSN : 1769-7298

Éditeur

UMR ESPACE

Référence électronique

Anne-Christine Bronner, « Le palmarès 2016 du concours Géovisualisation et cartographie dynamique », Mappemonde [En ligne], 120 | 2017, mis en ligne le 01 avril 2017, consulté le 15 septembre 2020. URL : http://journals.openedition.org/mappemonde/3166

Ce document a été généré automatiquement le 15 septembre 2020.

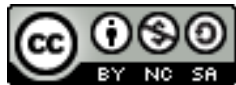

La revue Mappemonde est mise à disposition selon les termes de la Licence Creative Commons Attribution - Pas d'Utilisation Commerciale - Partage dans les Mêmes Conditions 4.0 International. 


\title{
Le palmarès $2016 \mathrm{du}$ concours Géovisualisation et cartographie dynamique
}

\author{
Anne-Christine Bronner
}

1 La neuvième édition du concours Géovisualisation et cartographies dynamiques (30 septembre-2 octobre 2016) a connu un beau succès avec 13 applications exposées au public et lors des mini-conférences du salon de la géomatique du festival international de géographie de Saint-Dié-des-Vosges.

2 Lors de cette édition, les jeunes générations ont été très présentes :

- avec les trois propositions du Master Géomatique appliquée aux études urbaines et aux risques de l'Université de Cergy-Pontoise : Mobi'Carto sur l'ofFre de transport, Mon école sous l'eau sur le risque d'inondation et une application sur le Harcèlement verbal envers les femmes;

- et plusieurs travaux de thèse, sur la cartographie des risques souterrains (M. Lacroix), l'analyse exploratoire des données spatio-temporelles (R. Cura) et la simulation de trafic routier (J. Richard).

3 La mise en œuvre du webmapping dans les collectivités a été illustrée de façon originale par la région Hauts-de-France avec un travail sur la valorisation par la carte des événements sportifs en prenant l'exemple du Paris-Roubaix.

4 L'IGN a concouru pour la première fois avec ses deux nouvelles applications : Minecraft à la carte et Remonter le temps.

5 La dimension internationale a été portée par plusieurs projets :

- la construction d'un réseau d'information géographique dans un contexte de développement local participatif dans un nouvel état indien « residual Andhra Pradesh»;

- une cartographie des toponymes de la ville Suisse de Carouge développé au sein de l'université de Genève ;

- la création d'un atlas numérique interactif sur l'Asie centrale, occidentale et le Caucase, incluant la dimension historique, CartOrient. 
Cette présentation permet de souligner la grande diversité des participants et des projets qui s'exposent dans le champs de la géovisualisation. Certains ont été développés sur la plateforme ArcGIS Online, alors que d'autres s'attachaient à s'inscrire dans le monde du libre (opendata, opensource). De nombreuses propositions ont été pointées pour des insuffisances dans la réflexion sur le plan de la représentation graphique, de la sémiologie.

\section{Les lauréats de l'édition 2016}

\section{Premier Prix}

7 Robin Cura

UMR Géographie-cités, LabEx DynamiTe \& Université Paris 1 Panthéon-Sorbonne

TimeLine Exploratory DashBoard

\section{Deuxième Prix}

8 François Lecordix

IGN - Institut national de l'information géographique et forestière

Minecraft à la carte

\section{Troisième Prix}

9 Nirbhay Sen, Bangaru Raju, Guru Prasad, Anne Chappuis (1), Sanjay Gupta (2) , Luc de Golbéry (consultant) (1) GMIS-Graphical Monitoring Information Systems Consultants Pvt Ltd., Hyderabad, India ; (2) APSDPS-Andhra Pradesh State Department of Planning and Statistics, Government of Andhra Pradesh

Building an interactive decentralized information system network for participative local centered development in a new indian state: "residual Andhra Pradesh"/Construire un système d'information participatif en appui au développement décentralisé dans le nouvel état "Andhra Pradesh» (Inde)

\section{Prix spécial catégorie Master}

10 Mélanie Vallui, Léo Brisot, Rémi Mayou, Laureline Gérard

Master Géomatique appliquée aux études urbaines et aux risques, Université de CergyPontoise

Mon école sous l'eau

Maxime Bricout, Pauline Etchart, Victor Le Gall

Master Géomatique appliquée aux études urbaines et aux risques, Université de CergyPontoise

Mobi'Carto

\section{Prix du public Mémoris}

11 Mélanie Vallui, Léo Brisot, Rémi Mayou, Laureline Gérard

Master Géomatique appliquée aux études urbaines et aux risques, Université de Cergy- 
Pontoise

Mon école sous l'eau

Retrouvez l'ensemble des applications présentées en 2016 et lors des différentes éditions sur le site du concours.

13 Composition du jury 2016:

-Anne-Christine Bronner, cartographe, laboratoire Sociétés, acteurs, gouvernement en Europe, CNRS-Université de Strasbourg

- Amandine Hamm, géomaticienne, Région Alsace Champagne-Ardenne Lorraine, site de Metz Olivier Klein, chercheur en géomatique, LISER, Luxembourg

- Yves Roynard, géomaticien, RATP, Paris

- Christophe Terrier, statisticien, Blog de veille statistique pour la géographie

- Marlène Villanova-Oliver, maître de conférences en informatique, Université Grenoble Alpes, Laboratoire Informatique de Grenoble, équipe STEAMER

14 Je remercie les partenaires qui soutiennent et permettent de récompenser tous les projets :

- l'ADFIG, association pour le développement du festival international de géographie

- DécryptaGéo, la revue indépendante de référence entièrement consacrée à l'information géographique

- Le laboratoire informatique de Grenoble

- Le Comité Français de Cartographie

- La MAIF

- Esri France, éditeur de solutions SIG

INDEX

Mots-clés : cartographie, cartographie dynamique, cartographie interactive, concours géovisualisation et cartographies dynamiques, FIG2016, géovisualisation

\section{AUTEUR}

\section{ANNE-CHRISTINE BRONNER}

Laboratoire SAGE - Sociétés, acteurs, gouvernement en Europe CNRS - Université de Strasbourg 